\title{
Vertical Micro Reactor Stack for Integrated Chemical Reaction System*
}

\author{
Toshifumi Asano, ${ }^{\dagger}$ Yoshiaki Ukita, and Yuichi Utsumi \\ Laboratory of Advanced Science and Technology for Industry, \\ University of Hyogo, 3-1-2, Koto, Kamigori, Ako-gun, Hyogo, 678-1205, Japan \\ Katsuhiro Matsui, Masahiro Takeo, and Seiji Negoro \\ Graduate School of Engineering, University of Hyogo, \\ 3-1-2, Koto, Kamigori, Ako-gun, Hyogo, 678-1205, Japan \\ (Received 13 May 2005; Accepted 26 May 2005; Published 10 June 2005)
}

\begin{abstract}
We proposed and fabricated vertical micro reactor stack with vertical fluid flow operation available for the environment analysis, post-genome analysis, gene diagnosis, and screenings of the useful materials for the medicine manufacture. This reactor is characterized as the simple structure and new aspects of the vertical fluid transportation evoked by the use of the fluid filter with micro through-bores. The LIGA process using synchrotron radiation was applied for the fabrication of the fluid filters. The CFD simulation results suggested that the fluid can be held by the fluid filter and easily transported by the pneumatic operation. It was also confirmed that the fluid flow velocity through the filter was controlled by varying the loaded pressure around several $\mathrm{kPa}$. Furthermore, it was expected that the fluid was stirred and mixed when passing through the fluid filter. It was demonstrated that a proposed chemical reactor had a good performance of the vertical fluid flow operation and chemical reaction.
\end{abstract}

[DOI: 10.1380/ejssnt.2005.190]

Keywords: Lithography; Biophysics, medical physics, and biomedical engineering; Solid-liquid interfaces; Micro reactor; Micro fluidics

\section{INTRODUCTION}

The advantages of micro reactors for the chemical synthesis, discovery and development of substances are generally recognized today. The significant properties of micro reactors such as low energy consumption, high speed, high yields, and short thermal response times for the chemical reactions will lead to the ecological and economical process engineering. Such properties result from the large surface area to volume ratios, rapid thermal diffusion, high gradients of pressure, and concentration of reagent solutions. The possibility to integrate a large number of micro reactors within a finite small space develop several major fields of application such as fine chemicals, combinatorial synthesis, high throughput screening using reactor units operated in parallel and in serial with different functions. However, the standard structure of the conventional micro rectors expands on two-dimensional substrate. From the point of micro integration and cost downing, such structure has a finite restriction. In order to solve above problems, 3D structured reactor operating by vertical fluid flow control have been expected so far. There are not so many examples for achieving such reactors. The fabrication of $3 \mathrm{D}$ structured reactor has several difficulty, since suitable microfabrication and packaging technique for vertical direction have not been well developed so far. Several attempts to fabricate stacked structures of 2D channel networks using vertical through holes and nano-porous membrane have performed in order to achieve 3D channel networks [1-4]. Ikuta et al also proposed the 3D stacked device so called gBiochem-

*This paper was presented at International Symposium on Molecule-Based Information Transmission and Reception - Application of Membrane Protein Biofuction- (MB-ITR2005), Okazaki, Japan, 3-7 March, 2005.

$\dagger$ Corresponding author: toshi@lasti.u-hyogo.ac.jp ical IC familyh using laser modeling technique [4]. These devices have been expected to utilize multiple fluid flows and miniaturize a total micro chemical system for micro combinatorial synthesis. We proposed and fabricated vertical micro reactor stack with vertical fluid flow operation available for the environment analysis, post-genome analysis, gene diagnosis, and screenings of the useful materials for the medicine manufacture. The reactor can operate vertically in stacked structures according to demanded chemical functions such as reaction, isolation, and purification. A variety of three-dimensional microfabrication processes brought by LIGA (abbreviated name of Lithographite, Galvanoformung and Abformung) process using synchrotron radiation allows new type of vertical micro reactors and their components. This reactor is characterized as the simple structure with no movable structure and new aspects of the vertical fluid transportation evoked by the use of the fluid filter with micro throughbores. We have designed the chemical reactor based on the results from the CFD (computational fluid dynamics) simulation and fabricated using LIGA process. Furthermore we investigated the vertical fluid transport properties and mixing property of the fluid filter. It is demonstrated that a proposed vertical micro reactor stack has a good performance of the vertical fluid flow operation.

\section{PROPOSAL OF VERTICAL MICRO REACTOR STACK WITH VERTICAL FLUID FLOW OPERATION}

In order to achieve the vertical fluid flow operation, we proposed the new method for vertical fluid flows as the fluid was transported through the filters with many micro through-bores. Figure 1 shows the schematic diagram for the stack of the proposed chemical reactor. As the figure shows the stack consists from two unit reservoirs and a liquid filter which separate them. The fluid in the upper reservoirs is held by surface tension of itself on the fluid fil- 


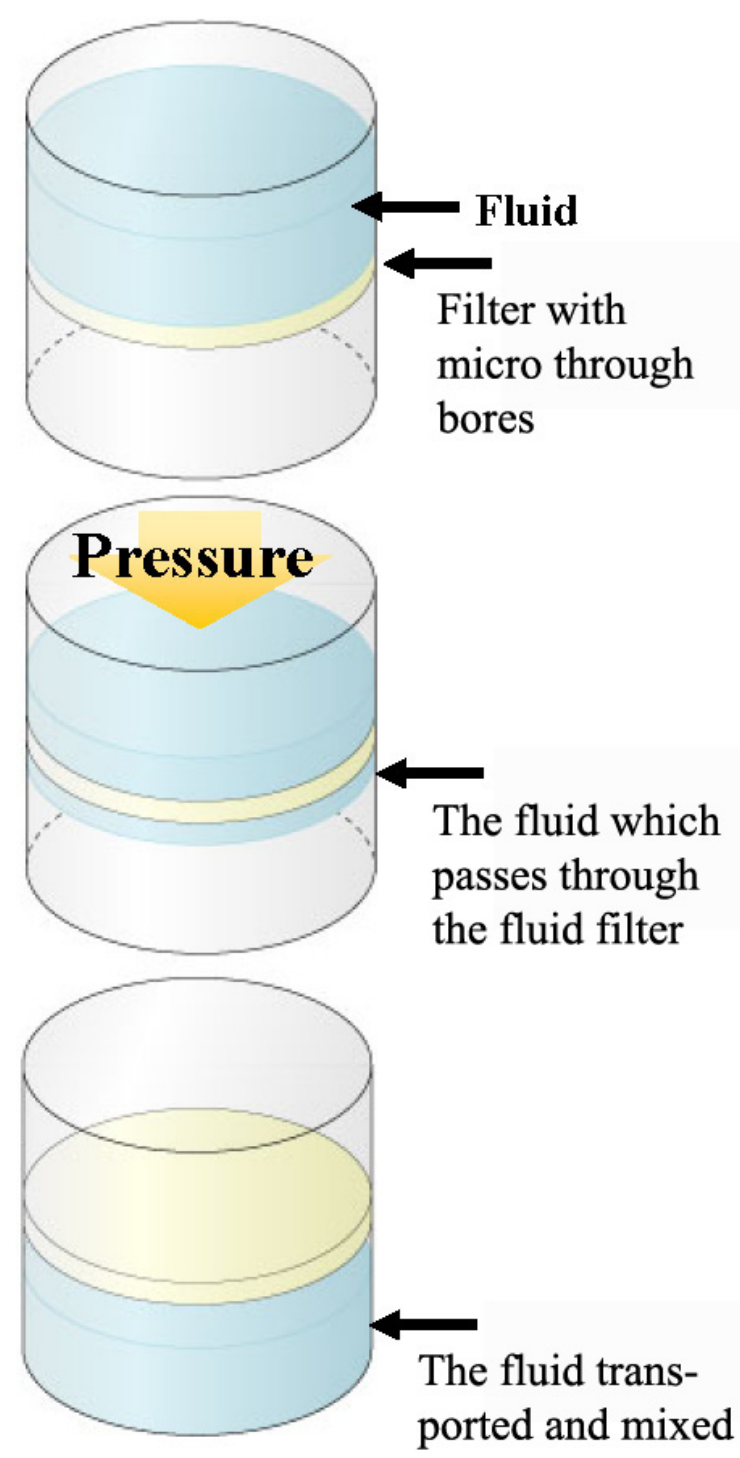

FIG. 1: Schematic diagram for the Vertical Fluid Flow Operation. (a) Without applying pressure, the fluid will be held by the fluid filter. (b) When applying the pressure, the fluid is passed through the fluid filter. (c) The fluid is stirred up and mixed drastically by the complex flow.

ters with through-bores. In the case of the transportation of the liquid from upper unit reservoir to the downward unit reservoir, the inside pressure of the upper reservoir is increased and transport the liquid through the filter to the downward reactor. It is prospective that the complex flow behavior when passing through the fluid filter will stir up the fluid drastically.

\section{DESIGN OF THE FILTER FOR VERTICAL FLUID FLOW OPERATION}

On the basis of the above vertical fluid transportation way we designed the chemical reactor in order to attain vertical unit chemical operation. The design is based on the results from the CFD (Computational Fluid Dynamics) simulation 'FLUENT'. In order to derive useful fluid flow properties, the effects of the structure parameters of

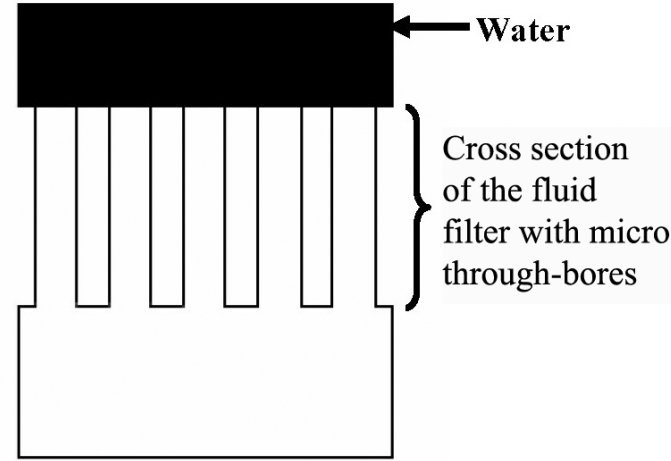

(a)

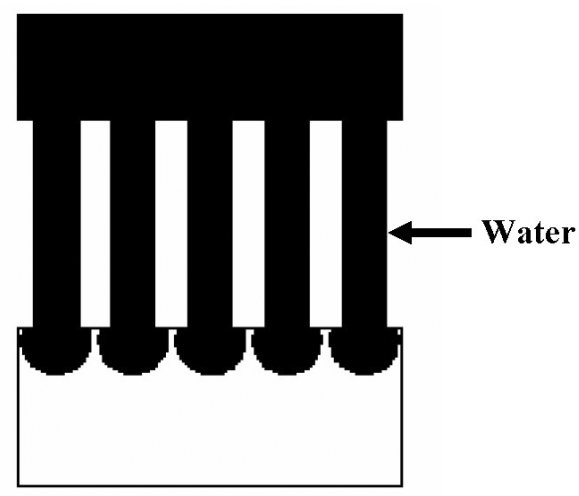

(b)

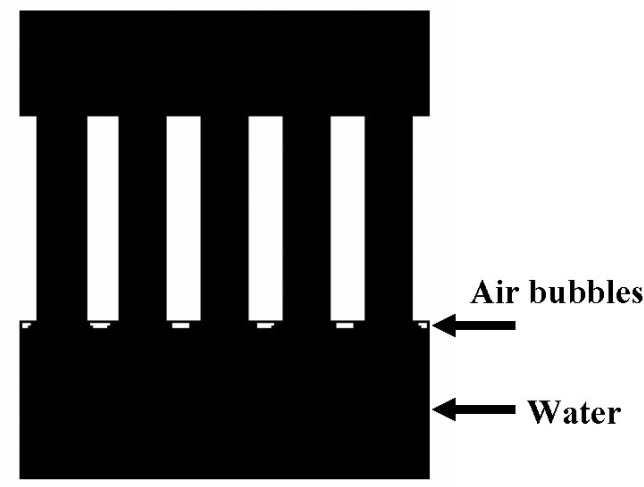

(c)

FIG. 2: Results of the CFD simulation, (a) holding of the water on the filter, passing time t0, (b) initial stage of the pneumatic transportation of the water to the downward reactor at $t=3.9 \times 10^{-4}$, (c) transportation of the water to the downward reactor at $t=1.08 \times 10^{-3}$.

the fluid filter on the fluid transportation were investigated. The material of the fluid filter was assumed to be PMMA or PTFE on the simulation. Figure 2 shows one of the results of the CFD simulation. The diameter and the thickness of the through-bores are $40 \mu \mathrm{m}$ and $200 \mu \mathrm{m}$. Material is assumed to be PTFE with the contact angle of $110^{\circ}$. The pneumatic pressure for the vertical fluid (water) operation is set at $3 \mathrm{kPa}$. As shown in Fig. 2(a)), the water is well sustained at the surface of the fluid filter. However, it is also found from the CFD results that if the radius of the through-bores become larger or the contact angle of the water become smaller the water is not able to be sustained anymore and the continuous water 


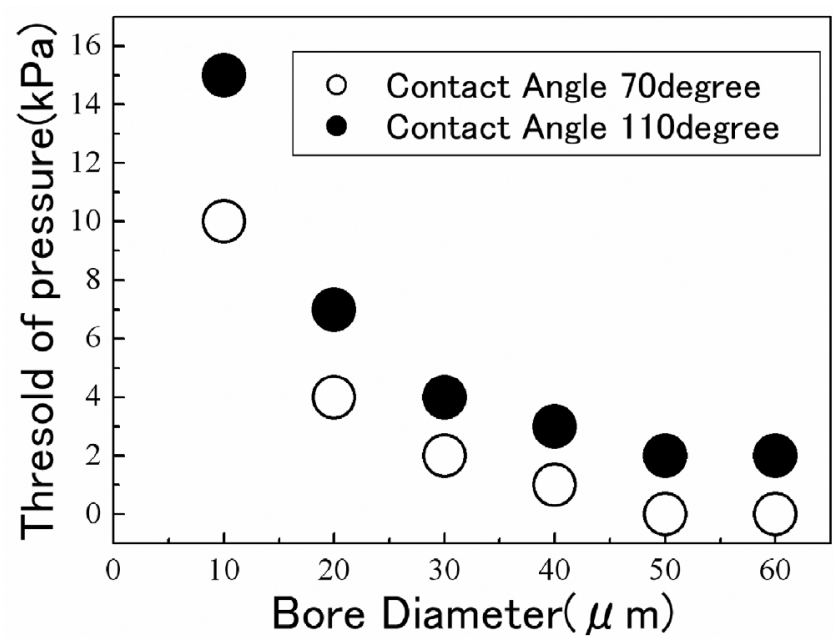

FIG. 3: Dependence of the threshold pressure on the bore diameter at which the pneumatic transportation of the fluid from the upward reservoir to the downward reservoir starts.

flow through the filter into downward reactor occurs. As shown in Fig. 2(b)), $3 \mathrm{kPa}$ pneumatic pressure is enough to transfer the water into downward reservoir. The passing time is estimated to be very short as $3.8 \times 10^{-4}$ sec. During the water flow through the filter, the air bubble is remained at the surface of the filter basal plain as shown in Fig. 2(c)). The affinity of the filter surface with water is also found to be very essential for sustaining of the water.

We estimated the dependence of the bore diameter on the threshold pressure at which the pneumatic transportation of the fluid from the upward reservoir to the downward reservoir starts by the use of the CFD simulation. We set the distance between the center of the bores and the contact angle of the water to be $70 \mu \mathrm{m}$ and $70^{\circ}$ or $110^{\circ}$, respectively. Figure 3 shows the result of the simulation. It shows hyperbolic distribution of the threshold and it increases with the bore diameter less than 30 $\mu \mathrm{m}$ significantly. It is notable that the threshold pressure for the pneumatic operation is fairly low compared with the atmosphere pressure and suggests the pressure loss of the vertical fluid transportation at the reactor stack with several layers restricted to be low less than quarter atom. From the easiness of the control for the pneumatic pressure driving vertical liquid transportation, the smaller bore diameter is desirable since the margin of the pressure set for the vertical flow transportation becomes broader. However because of the limitation of our micro fabrication ability, the smallest bore diameter restricted to $20 \mu \mathrm{m}$ in the $400 \mu \mathrm{m}$ thick film structure.

\section{FABRICATION PROCEDURE OF THE REACTOR USING SYNCHROTRON RADIATION}

After the above design based on the computer aided fluidic dymamics simulation, the chemical reactor stacks which consist from two or three unit vessels were fabricated using the X-ray lithography and the high precision mechanical fabrication. The shape of the unit vessel was cylinder of which diameter and height were $3 \mathrm{~mm}$ and 11 $\mathrm{mm}$ respectively. The reactor has the volume of sereral decaded micro litters suitable for the biochemical analysis using test tubes. The micro liquid filters with cylindrical through-bores were fabricated using deep X-ray lithography by irradiating synchrotron radiation to $200 \mu \mathrm{m}$ or $400 \mu \mathrm{m}$ thick PMMA sheets. The diameter of the bores ranged from 40 to $50 \mu \mathrm{m}$. The deep X-ray exposures were performed using LIGA beamline (BL2) at NewSUBARU [5-7] which was established at Hyogo, Japan. The X-ray masks with $10 \mu \mathrm{m} \mathrm{Au}$ absorbers were manufactured in order to obtain high aspect ratio micro bores. Figure 4(a) shows the SEM images of the liquid filter with $400 \mu \mathrm{m}$ thickness. Figure 4(b) shows the outward appearance of the reactor which consists from three reservoirs.

\section{EVALUATION OF THE FLUID TRANSPORTATION PROPERTY}

The vertical fluid flow through the micro filter is a key function for this reactor. We evaluated the property of the fluid flow by loading the positive air pressure insides the upper unit reservoir and measuring the flow velocity as a function of the loading pressure. We adopted the pure water as the fluid in the reactor and demonstrated the vertical fluid flow from the upper reservoir unit to the lower reservoir unit. First of all the vertical operation is confirmed by observing both of the sustaining of the fluid and the following transportation to downward reservoir driven by the pneumatic pressure.

Next we investigated the property of the fluid transportation from upper reservoir to the downward reservoir by measuring the passing time of the fluid through the filter. The dependence of the pneumatic flow rate on the water flow rate was investigated. Figure 5 shows the dependences of the water flow rate on the pneumatic flow rate with various loaded pressure from 5 to $10 \mathrm{kPa}$ when using the filter with $40 \mu \mathrm{m}$ bore diameter and $200 \mu \mathrm{m}$ thickness. The figure shows that the dependence of the fluid flow transportation behavior through the filter on the loaded pneumatic pressure is not significant at the 40 $\mu \mathrm{m}$ bore diameter of the filter. The threshold can not be found for the pneumatic flow rate at which vertical fluid flow through the fluid filter starts. The results also suggest that the flow velocity can be easily controlled by varying the loaded pressure.

\section{MIXING PROPERTY OF THE FLUID FILTER}

We tried to conduct an enzyme reaction by using this reactor and the reaction was observed optically in the reservoir in real time. The enzyme reaction produces the 2-hydroxymuconate semialdehyde, which has characteristic absorption at the wavelength of 375 nm. The absorbance depends on the amount of the 2hydroxymuconate semialdehyde. So we can detect the process of the reaction by observing the absorbance in real time.

First of all, the blending of enzyme (catechol 2,3dioxygenase) and matrix (catechol) liquid was done. They 


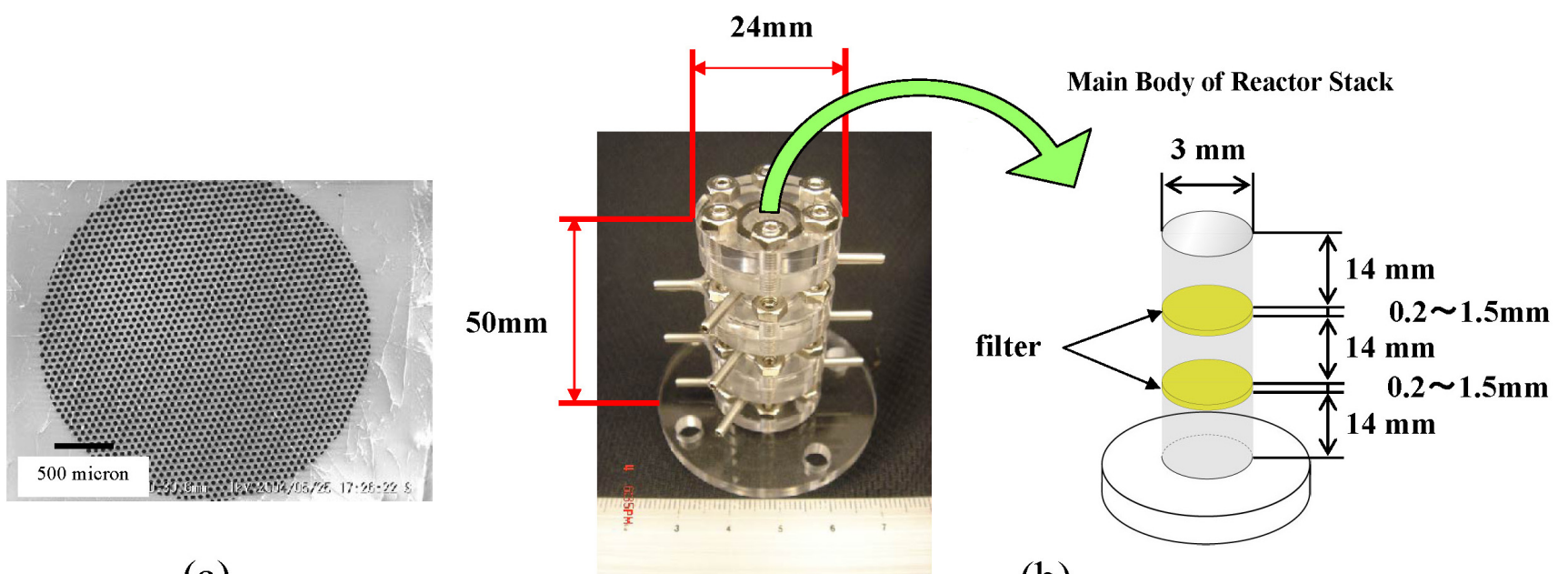

(a)

(b)

FIG. 4: (a) The SEM image of the micro fluid filters with round through-bores. (b) Outward appearance of the reactor which consists of three reservoirs.

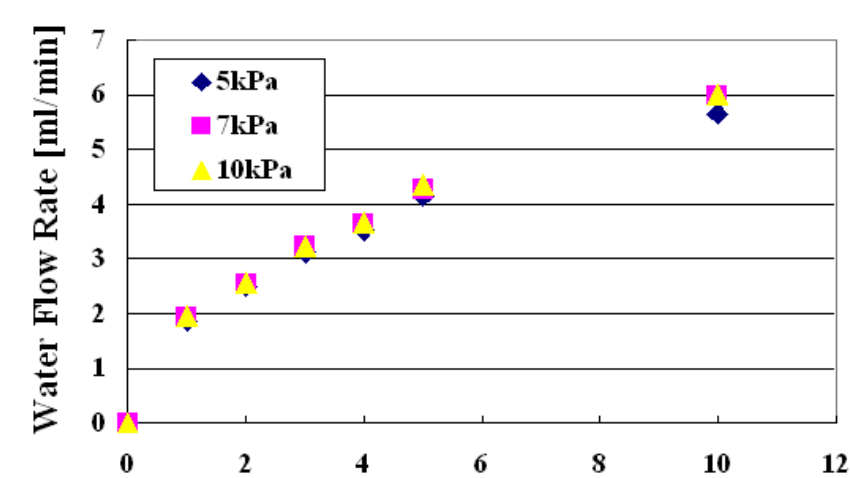

Pneumatic Flow Rate $[\mathrm{ml} / \mathrm{min}]$

FIG. 5: The measured water transportation property of the fluid filter with the $40 \mu \mathrm{m}$ bore diameter and the $200 \mu \mathrm{m}$ thickness for the three loaded pneumatic pressure from $5 \mathrm{kPa}$ to10 $\mathrm{kPa}$ are shown.

are injected into upper reservoir and mixed. The enzyme reaction occurs through this operation. Then, the mixture was transported by the pneumatic pressure to downwards. The absorbance of the 2-hydroxymuconate semialdehyde was measured to detect the reaction. Figure 6 is the schematic diagram of the applied enzyme reaction and the observed time shift of the absorbance at wavelength of $375 \mathrm{~nm}$.

The figure shows shortening of reactive time after passing of the fluid filter. It is speculated that the increase of reactive efficiency results from the mixing of reagents during their passing through the fluid filter.

\section{CONCLUSION}

We proposed and fabricated vertical micro reactor stack with vertical fluid flow operation available for the environment analysis, post-genome analysis, gene diagnosis, and screenings of the useful materials for medicine manufac-

\section{Catechol 2,3-dioxygenase}<smiles>CI(I)CC(/C=C\C=O)=C(\O)C(=O)O</smiles>

\section{Catechol 2-hydroxymuconate} semialdehyde

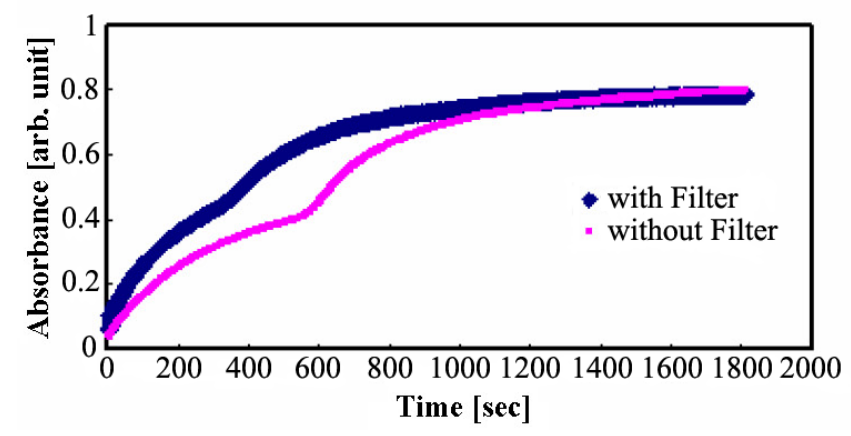

FIG. 6: The result of the reactive characteristics with fluid which passed through the filter and without filter.

tures. This reactor is characterized as the simple structure and new aspects of the vertical fluid transportation evoked by the use of the fluid filter with micro throughbores. The LIGA process using synchrotron radiation was used for the fabrication of the fluid filters for vertical fluid operation. The experiments showed that the water could be sustained on the surface of the fluid filter we proposed. We investigated the property of the fluid transportation from upper reservoir to the downward reservoir by measuring the passing time of the fluid through the filter. The five $\mathrm{kPa}$ pneumatic pressure is enough to transform the water into downward reservoir. The affinity of the 
filter surface to the water is found to be very essential for sustaining of the water. The results also suggest that the flow velocity can be easily controlled by varying the loaded pressure. It is demonstrated that a proposed vertical micro reactor stack has a good performance of the vertical fluid flow operation. The fluid is thought to be mixed by passing through the filter, and shortening of reactive time and the improvement of the reactive efficiency are expected.
[1] B. H. van der Schoot, S. Jeanneret, A. van den Berg and N.F. de Rooij, Sensors and Actuators B 15-16, 211 (1993).

[2] T. C. Kuo, D. M. Cannon, W. Feng, M. A. Shannon, J. V. Sweedler, and P. W. Bohn, Proc. Micro Total Analysis Systems 2001, 60 (2001).

[3] Y. Kikutani, H. Hisamoto, M. Tokeshi, and T. Kitamori, Proc. Micro Total Analysis Systems 2001, 161 (2001).

[4] K. Ikuta, S. Maruo, T. Fujisawa, and A.Yamada. MEMS99, pp. 376-381 (1999).
[5] A. Ando, S. Amano, S. Hashimoto, H. Kinoshita, S. Miyamoto, T. Mochizuki, M. Niibe, Y. Shoji, M. Terasawa, T. Watanabe, and N. Kumagai, J Synchrotron Rad. 5, 342 (1998).

[6] Y. Utsumi, T. Kishimoto, H. Mekaru, and T. Hattori, Proc.ICEE 2004, pp. 608-613 (2004).

[7] Y. Utsumi, T. Kishimoto, H. Mekaru, and H. Hara, Proc. IWMF 2004, pp. 275-280 (2004). 